\title{
Dielectrophoretic analysis of changes in cytoplasmic ion levels due to ion channel blocker action reveals underlying differences between drug-sensitive and multidrug-resistant leukaemic cells
}

L Duncan $^{1}$, H Shelmerdine ${ }^{1}$, MP Hughes ${ }^{1}$, HM Coley $^{2}$, Y Hübner ${ }^{1}$, and FH Labeed ${ }^{1} *$

${ }^{1}$ Centre for Biomedical Engineering, School of Engineering (H5), University of Surrey, Guildford, GU2 7XH, UK

${ }^{2}$ Postgraduate Medical School, University of Surrey, Guildford, GU2 7XH

*E-mail: f.labeed@surrey.ac.uk 


\begin{abstract}
Dielectrophoresis - the motion of particles in non-uniform AC fields - has been used in the investigation of cell electrophysiology. The technique offers the advantages of rapid determination of the conductance and capacitance of membrane and cytoplasm. However, it is unable to directly determine the ionic strengths of individual cytoplasmic ions, which has potentially limited its application in assessing cell composition. In this paper, we demonstrate how dielectrophoresis can be used to investigate the cytoplasmic ion composition by using ion channel blocking agents. By blocking key ion transporters individually, it is possible to determine their overall contribution to the free ions in the cytoplasm. We use this technique to evaluate the relative contributions of chloride, potassium and calcium ions to the cytoplasmic conductivities of drug sensitive and resistant myelogenous leukaemic (K562) cells in order to determine the contributions of individual ion channel activity in mediating multi-drug resistance in cancer. Results indicate that whilst $\mathrm{K}^{+}$and $\mathrm{Ca}^{2+}$ levels were extremely similar between sensitive and resistant lines, levels of $\mathrm{Cl}^{-}$were elevated by three times that in the resistant line, implying increased chloride channel activity. This result is in line with current theories of MDR, and validates the use of ion channel blockers with DEP to investigate ion channel function.
\end{abstract}




\section{INTRODUCTION}

Dielectrophoresis (DEP) is a phenomenon of induced particle motion on the micro- and nanoscale, with a broad range of applications to medicine (Pohl 1978, Hughes 2002). It belongs to a family of electrical phenomena that includes the more commonly used technique of electrophoresis, but is functionally different in two aspects. First, it is more readily amenable for use with whole cells; second, it uses an alternating (rather than constant) energizing voltage whose frequency is swept over a wide range, producing a spectrum that allows the analyst to determine the electrical properties (typically capacitance and electrical conductivity) of the membrane and cytoplasm (rather than merely the net particle charge). Furthermore, DEP is label-free and non-invasive, relying on the measurement of cell motion in nonuniform electric fields to infer the electrical properties. However, it is unable to directly determine the ionic strengths of individual cytoplasmic ions, which has potentially limited its application in assessing cell composition; in this paper, we demonstrate how dielectrophoresis can be used to investigate the cytoplasmic ion composition by using ion channel blocking agents. Our previous work (Labeed et al 2003) has shown that drug sensitive (K562) and doxorubicin resistant leukaemic (K562AR) cancer cells exhibited significant differences in their dielectric properties, most notably in the conductivity (related to free ions) of the cytoplasm. K562 cells exhibited lower cytoplasmic conductivity- ionic strength relative to K562AR. Broader studies (Coley et al 2007) have indicated that there is a direct correlation between drug resistance and cytoplasmic conductivity.

Cancer cell resistance to chemotherapeutic agents can present a serious impediment to the successful treatment of cancer and is responsible for many deaths each year. One form of drug resistance is called multidrug resistance (MDR), characterised by cross-resistance to many anti-cancer drugs that have no common structure - but which are notably natural product in origin. Several mechanisms have been attributed to the MDR phenotype. A common correlate of MDR is the over-expression of membrane glycoproteins, collectively termed ABC (ATP-binding-cassette) transporters. Specifically, the MDRl gene product P-glycoprotein pump (P-gp) has been closely associated with MDR status in cells. However, 
there is no clear consensus regarding the way in which P-gp confers drug resistance; rather, two models of MDR have been proposed, based on different aspects of P-gp activity. It has been postulated to play a direct role in drug efflux, "pumping" drugs (which act as substrates for the pump) from the membrane and giving rise to reduced and ineffective intracellular drug concentrations (Juliano and Ling 1976, Gottesman et al 2002). However, P-gp has also been reported to be a $\mathrm{Cl}^{-}$channel (Valverde et al 1992, Wadkins et al 1997) or bifunctional with one function as a $\mathrm{Cl}^{-}$channel and another as a peptide transporter (Gill et al 1992). It has also been noted that P-gp shares homology with the cystic fibrosis transmembrane conductance regulator (CFTR) (Welsh et al 1992, Gadsby et al 1999), a known $\mathrm{Cl}^{-}$channel.

In this paper, we have used DEP to determine the relative contributions of chloride, potassium and calcium ions to the cytoplasmic conductivity of drug-sensitive and drug-resistant (P-gp expressing) leukaemic cells in order to determine the contributions of individual ion channel activity in mediating multi-drug resistance in cancer. K562 and K562AR cells were separately cultured with agents to specifically block calcium, chloride and potassium (verapamil, NPPB and quinine, respectively). This allowed a determination of the contribution of each ion species to the net conductivity of the untreated cell and, hence, showed the quantity of specific ion species present in the cells. Results indicate that whilst $\mathrm{K}^{+}$ and $\mathrm{Ca}^{2+}$ levels were extremely similar between sensitive and resistant line, levels of $\mathrm{Cl}^{-}$were elevated by up to three times in the resistant line, implying increased chloride channel activity that can be associated with P-gp overexpression and supports the notion of P-gp as a chloride channel. However, treatment using the MDR modulator XR9576 (Roe et al 1999) showed no change in cytoplasmic conductivity despite reversing drug resistance. This correlates with the dual-function role of P-gp (Wadkins et al 1997) and indicates that its function as a chloride transporter plays no significant role in conferring MDR status in cells. 


\section{THEORY}

A dielectrophoretic force occurs due to the interaction of an induced dipole and an electric field gradient. The time averaged force $\mathbf{F}_{\text {DEP }}$ on a homogeneous dielectric sphere, (Hughes 2002) is:

$$
\mathbf{F}_{\mathrm{DEP}}=\operatorname{Re}[K(\omega)] 2 \pi_{r}^{3} \varepsilon_{m} \nabla \mathbf{E}_{r m s}^{2}
$$

where $r$ is the particle radius, $\varepsilon_{m}$ is the absolute permittivity of the suspending medium, $\nabla$ the del vector operator, $\mathbf{E}_{r m s}$ is the local RMS electric field and $\operatorname{Re}[K(\omega)]$ is the real part of the Clausius-Mossotti factor, given by:

$$
K(\omega)=\frac{\varepsilon_{p}^{*}-\varepsilon_{m}^{*}}{\varepsilon_{p}^{*}+2 \varepsilon_{m}^{*}}
$$

where $\varepsilon_{m}{ }^{*}$ and $\varepsilon_{p}{ }^{*}$ are the complex permittivities of the medium and particle respectively. A general permittivity is defined as $\varepsilon^{*}=\varepsilon-j \frac{\sigma}{\omega}$ with permittivity $\varepsilon$ and conductivity $\sigma$. The direction of the force (positive or negative DEP) is governed by the value of $\operatorname{Re}[K(\omega)]$, which can take positive or negative values respectively depending on the applied frequency or the particle and suspending medium conductivity and permittivity. We can adapt this for a more complex sphere such as a cell, where the inner cytoplasm is enclosed by an enveloping membrane. If we consider the cytoplasm as being compartment 1 and having radius $a_{l}$ surrounded by the membrane which we consider as compartment 2 and have radius $a_{2}$, then we can replace the complex permittivity of the particle $\varepsilon_{p}{ }^{*}$ with an effective particle permittivity $\varepsilon^{*}{ }_{\text {leff }}$ formed from the combined properties of membrane and cytoplasm, thus (Hughes 2002): 


$$
\varepsilon_{1 e f f}^{*}=\varepsilon_{2}^{*} \frac{\left(\frac{a_{2}}{a_{1}}\right)^{3}+2 \frac{\varepsilon_{1}^{*}-\varepsilon_{2}^{*}}{\varepsilon_{1}^{*}+2 \varepsilon_{2}^{*}}}{\left(\frac{a_{2}}{a_{1}}\right)^{3}-\frac{\varepsilon_{1}^{*}-\varepsilon_{2}^{*}}{\varepsilon_{1}^{*}+2 \varepsilon_{2}^{*}}}
$$

This value provides an expression for the combined complex permittivity of the particle at any given frequency $\omega$. It can also be combined with the complex permittivity of the medium to calculate the Clausius-Mossotti factor.

We propose that by using this model in conjunction with ion channel blockers, it is possible to determine the relative contribution of major ion species in the cytoplasm. The cytoplasmic conductivity is the net product of the mobile ionic species contained within the cytoplasm; when the cell membrane is allowed to permeabilise, the cytoplasmic conductivity drops to single-mS values (Hübner et al 2005). We hypothesise that if ion channel-blocking agents are used, the cytoplasmic conductivity will drop due to the absence of the corresponding ion species in the cytoplasm; if a range of ion channel blockers is used and key ion species are removed one at a time, then by simple algebra it should be possible to determine the actual contribution of each ion species to the total conductivity, and hence to determine the relative concentration of each ion species in the cytoplasm of the cells.

\section{MATERIALS AND METHODS}

\subsection{Chemicals and reagents}

Doxorubicin was dissolved in sterile distilled water and stored frozen as stock solutions, which were thawed prior to use. The 5-Nitro-2-(3-phenylpropylamino) benzoic acid (NPPB) was dissolved in dimethylsulfoxide, and water was used for quinine and verapamil. All reagents were purchased from Sigma Aldrich, Poole, UK. XR9576 was prepared as described in detail by Labeed et al (2003). 


\subsection{Cell culture and ion channel blockers}

Human chronic myelogenous leukaemia (K562), and its doxorubicin resistant counterpart (K562AR), were grown in 20 mM HEPES modified RPMI-1640 medium supplemented with $10 \%$ heatinactivated foetal calf serum (FCS), (Invitrogen, Paisley, UK), $2 \mathrm{mM} \mathrm{L-} \mathrm{glutamine} \mathrm{and} 100$ units/ mL penicillin-streptomycin. All cell culture reagents were obtained from Sigma Aldrich (Poole, UK), unless stated otherwise. The cells were grown under standard cell culture conditions with $5 \% \mathrm{CO}_{2} / 95 \%$ air at $37^{\circ} \mathrm{C}$. K562AR were maintained in the presence of $100 \mathrm{nM}$ doxorubicin which was removed at least 1 passage prior to use of cells in experiments.

A density of $3 \times 10^{5}$ cells per $\mathrm{mL}$ was used for ion channel blocker treatments, and the cells were incubated for two hours prior to DEP analysis. This procedure was followed for each agent: $20 \mu \mathrm{M}$ verapamil, 10 $\mu \mathrm{M}$ NPPB and $0.1 \mathrm{mM}$ quinine, 100nM XR9576.

The MDR status of K562AR has been confirmed previously (data not shown, for similar results see previous work Labeed et al 2003) using western immunoblotting and MTT-cytotoxicity testing. These previous analyses confirmed the overexpression of P-gp, for K562AR cells, whilst cytotoxicity testing showed them to be 14 fold resistant to doxorubicin relative to K562 parental cells.

\subsection{DEP experiments}

The drug resistant and sensitive cells were centrifuged at room temperature at $190 \times \mathrm{g}$ for 5 minutes. The pellets were washed and resuspended in iso-osmotic medium consisting of $8.5 \%(\mathrm{w} / \mathrm{v})$ sucrose plus $0.3 \%$ (w/v) dextrose buffer (Gascoyne et al 1997). The medium conductivity was adjusted to $2.5 \mathrm{mS} / \mathrm{m}$ using PBS and the final conductivity, before use, was verified with a conductivity meter (RS components Ltd, London, UK). The final cell population was counted using a haemocytometer and adjusted to approximately $3 \times 10^{5}$ cells/ $\mathrm{mL}( \pm 15 \%)$ for DEP measurements. In order to reduce the effect of variation in cell number in each sample, the experiments were repeated many times (generally 4-6) with different populations, which were summed prior to modelling. 
The dielectric properties of the cells, after each ion channel blocker were determined by fitting the measurement spectra to the single shell model (Broche et al 2005). The best fit model was found by matching the curve to the measured data, and then altering the dielectric parameters of the membrane and the cytoplasm until a best match was found. For DEP calculations, cell diameters were measured using Photolite software to analyse microscope images of cells.

\section{RESULTS AND DISCUSSION}

DEP spectra were taken for K562 and K562AR before and after treatment with ion channel blockers, and the dielectric properties of the cells were extracted using principles outlined by Broche et al. (2005), with particular attention paid to the conductivity of the cytoplasm $\left(\sigma_{\text {cyto }}\right)$. This parameter represents the conduction of free ions through the cytoplasm, and is composed of the sum of the contributions of the ion species present. The values of cytoplasmic conductivity for control cells and those having been incubated with the blockers, plus data for cells incubated with XR9576, are shown in Table 1.

The results showed significant differences in the cytoplasm of the drug sensitive K562 relative to drug resistant counterparts. This difference has already been described in the literature (Labeed et al 2003) but its origin has not been discussed. As shown in Table 1, untreated control K562 cells exhibit a lower $\sigma_{\text {cyto }}(0.28 \mathrm{~S} / \mathrm{m})$ relative to the control drug resistant counterpart $(0.50 \mathrm{~S} / \mathrm{m})$. Treating both cell lines with verapamil (a calcium channel blocker) and quinine (a potassium channel blocker) caused approximately the same degree of reduction in the $\sigma_{\text {cyto }}$ (approximately $-0.1 \mathrm{~S} / \mathrm{m}$ and $-0.15 \mathrm{~S} / \mathrm{m}$ respectively). However, using NPPB (a chloride channel blocker) resulted in significantly different changes in the two lines, with both cell lines exhibiting the same $\sigma_{\text {cyto }}(0.25 \mathrm{~S} / \mathrm{m})$ values after treatment. Notably, treating either cell line with XR9576 produced no significant change in the value of $\sigma_{\text {cyto }}$.

In order to understand these results, it is necessary to make an approximation. We suggest that we can consider the cell to be composed of three primary conducting agents $-\mathrm{K}^{+}, \mathrm{Ca}^{2+}$ and $\mathrm{Cl}^{-}$- plus some 
minor contribution from other charged species (DNA and proteins), whose value (observed in cells that have lost membrane integrity) can be at least an order of magnitude less than for viable cells (Labeed et al 2006). Assuming that each blocker selectively removes one ion species from this list, a simultaneous equation can be constructed to determine the contribution each ion makes to the total value of cytoplasmic conductivity. We emphasise that this process is an approximation, since factors such as conductivity change due to water loss (Labeed et al 2006) are not considered - there was no significant change observed in the radii of K562 cells after treatment, but some shrinkage was seen in blocker-treated K562AR - but the results do highlight trends in the ionic composition of the two species.

Considering the results, we found that a good match was found to the data for ionic contributions for $\mathrm{K}^{+}, \mathrm{Ca}^{2+}$ and $\mathrm{Cl}^{-}$of $0.13 \mathrm{Sm}^{-1}, 0.08 \mathrm{Sm}^{-1}$ and $0.09 \mathrm{Sm}^{-1}$ for $\mathrm{K} 562$, and $0.16,0.08$ and $0.25 \mathrm{Sm}^{-1}$ for K562AR. Assuming an error margin of $10 \%$, it is evident that for $\mathrm{K}^{+}$and $\mathrm{Ca}^{2+}$ the values are sufficiently similar to suggest that they are the same between the two lines; however, the data suggest there is a significant (three-fold) difference in cellular chloride levels between parent and resistant lines.

This result is significant for two reasons. First, it indicates that the primary electrophysiological difference in the two lines can be attributed to the presence of additional chloride, suggesting either an increase in activity of chloride channels in the resistant line. This strongly correlates with the notion that P-gp is a chloride channel. However, it is also important to note that the MDR modulator XR9576 does not change the electrical properties of the cell - that is to say, the drug resistance of the cell is not affected by the chloride channel activity of P-gp, but by another mechanism which inhibits resistance without affecting the chloride channel function of the P-gp molecule.

In summary, we have demonstrated that the use of ion channel blockers allows the approximate determination of cytoplasmic ionic composition, and hence the quantify the activity of ion channels noninvasively in large numbers of cells simultaneously. The results indicate that the membrane protein P-gp does act as a chloride channel, but that this function is neither correlated with the conferring of MDR status, nor deactivated by the inhibition of P-gp in resistance modulation. . 


\section{AKNOWLEDGEMENTS}

We thank the Nuffield Bursary scheme for funding HS, and Xenova PLC (Millenium) for the supply of XR9576.

\section{REFERENCES}

Broche L, Labeed F H and Hughes M P 2005 Extraction of dielectric properties for multiple populations from dielectrophoretic collection spectrum data, Phys. Med. Biol. 50 2267-74.

Coley H M, Labeed F H, Thomas H and Hughes M P 2007 Biophysical characterization of multidrug resistance in MCF-7 cell lines reveals the cytoplasm is a critical factor determining drug sensitivity Biochim. Biophys. Acta, 1770 601-08.

Gadsby D C and Nairn A C 1999 Regulation of CFTR $\mathrm{Cl}^{-}$ion channels by phosphorylation and dephosphorylation Adv. Sec. Mess. Phosph. 33 79-106.

Gascoyne P R C, Wang X B, Huang Y and Becker F F 1997 Dielectrophoretic separation of cancer cells from blood, IEEE Trans. Ind. Appl. 33 670-78.

Gill D R, Hyde S C, Higgins C F, Valverde M A, Mintenig G M and Sepulveda F V 1992 Separation of drug transport and chloride channel functions of the human multidrug resistance P-glycoprotein Cell 71 23-32.

Gottesman M M, Fojo T and Bates S E 2002 Multidrug resistance in cancer; role of ATP-dependent transporters Nature Rev. Cancer 2 48-58.

Hübner Y, Hoettges K F, Kass G E N, Ogin S L and Hughes M P 2005 Parallel measurements of drug actions on Erythrocytes by dielectrophoresis, using a novel 3-dimensional electrode design IEE Proc. Nanobiotech. 4 21-25

Hughes M P 2002 Nanoelectromechanics in Engineering and Biology, CRC Press, Boca Raton. Juliano R L and Ling V A 1976 A surface glycoprotein modulating drug permeability in Chinese hamster ovary cell mutants Biochim. Biophys. Acta 255 152-62. 
Labeed F H, Coley H M, Thomas H and Hughes M P 2003 Assessment of multidrug resistance reversal using dielectrophoresis and flow cytometry, Biophys. J. 85 2028-34.

Labeed F H, Coley H M and Hughes M P 2006 Changes in the cytoplasm of K562 myelogeneous leukaemic cancer cells during apoptosis, observed in real time by dielectrophoresis, Biochim. Biophys. Acta. 1760 922-29.

Pohl H A 1978 Dielectrophoresis, Cambridge University Press, Cambridge.

Valverde M A, Diaz M, Sepulveda F V, Gill D R, Hyde S C and Higgins C F 1992 Volume-regulated chloride channels associated with the human multidrug-resistance P-glycoprotein Nature 355 830-33.

Wadkins R M and Roepe P D 1997 Biophysical aspects of P-glycoprotein-mediated multidrug resistance Int. Rev. Cytol. 171 121-65.

Welsh M J, Anderson M P, Rich D P, Berger H A, Denning G M, Ostedgaard L S, Sheppard D N, Cheng S H, Gregory R J and Smith A E 1992 CFTR: a chloride channel with novel regulation Neuron 8 821-29. 


\begin{tabular}{lc}
\hline \multicolumn{1}{c}{ Cell Line } & $\boldsymbol{\sigma}_{\text {cyto }}(\mathbf{S} / \mathbf{m})$ \\
\hline K562 & \\
-control & 0.28 \\
-with verapamil & 0.22 \\
-with quinine & 0.27 \\
-with NPPB & 0.25 \\
-with XR9576 & 0.28 \\
K562AR & \\
-control & 0.50 \\
-with verapamil & 0.41 \\
-with quinine & 0.34 \\
-with NPPB & 0.25 \\
-with XR9576 & 0.55 \\
\hline
\end{tabular}

Table 1: A table summarizing the values of cytoplasmic conductivity measured after using ion channel blockers in K562 and K562AR, plus controls and cells cultured in the presence of MDR modulator XR9576. All results fit subject to an error margin of $10 \%$ or less. 